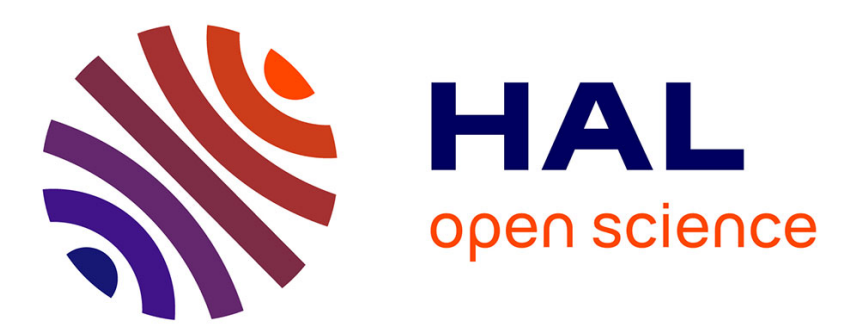

\title{
Asymptotic distribution of circularity coefficients estimate of complex random variables
}

Jean-Pierre Delmas, Habti Abeida

\section{To cite this version:}

Jean-Pierre Delmas, Habti Abeida. Asymptotic distribution of circularity coefficients estimate of complex random variables. Signal Processing, 2009, 89 (12), pp.2670-2675. 10.1016/j.sigpro.2009.05.013 . hal-00444484v2

\section{HAL Id: hal-00444484 \\ https://hal.science/hal-00444484v2}

Submitted on 30 May 2016

HAL is a multi-disciplinary open access archive for the deposit and dissemination of scientific research documents, whether they are published or not. The documents may come from teaching and research institutions in France or abroad, or from public or private research centers.
L'archive ouverte pluridisciplinaire HAL, est destinée au dépôt et à la diffusion de documents scientifiques de niveau recherche, publiés ou non, émanant des établissements d'enseignement et de recherche français ou étrangers, des laboratoires publics ou privés. 


\title{
Asymptotic distribution of circularity coefficients estimate of complex random variables
}

\author{
Jean Pierre Delmas and Habti Abeida
}

\begin{abstract}
In this correspondence we mainly consider the asymptotic distribution of the estimator of circularity coefficients of scalar and multidimensional complex random variables (RV). A particular attention is paid to rectilinear RV. After deriving new properties of the circularity coefficients, the maximum likelihood estimate of the circularity coefficients in the Gaussian case and asymptotic distribution of this estimate for arbitrary distributions are given. Finally, an illustrative example is presented in order to strengthen the obtained theoretical results.
\end{abstract}

Keywords: Circular/noncircular, proper/improper, rectilinear signal, coherence matrix, canonical correlations, circularity spectrum, circularity coefficients.

\section{INTRODUCTION}

Many papers (see, e.g., [1], [2], [3]) show that significant performance gains can be achieved by the secondorder algorithms exploiting the statistical information contained in the complementary covariance [4] matrix $\mathbf{R}_{z}^{\prime}=\mathrm{E}\left(\mathbf{z z}^{T}\right)$ provided it is non-zero (also termed as relation matrix in [5] and pseudo covariance matrix in [6]) in addition to that contained in the standard covariance matrix $\mathbf{R}_{z}=\mathrm{E}\left(\mathbf{z z}^{H}\right)$. Signals such that $\mathbf{R}_{z}^{\prime} \neq \mathbf{O}$, referred to as improper [4] or second-order non-circular [5], [6], occur in many signal processing applications. Particularly many digital modulations of practical interest, such as BPSK, M-ASK, OQPSK MSK and GMSK contain improper processes. But these signals have received a resurgence of interest. To assess detection or estimation performance of algorithms adapted to improper signals, the circularity spectrum, whose elements are referred to as circularity coefficients in [6] and fruitfully interpreted as canonical correlations between $\mathbf{z}$ and $\mathbf{z}^{*}$ simultaneously and independently introduced in [7], has been introduced. These parameters have been studied from different points of view. They allow one to specify conditions for identifiability, separability and uniqueness in complex-valued independent component analysis in [6]. They are used to design a generalized 
likelihood ratio test (GLRT) for impropriety in [7] through a specific measure for the degree of impropriety. Different measures of this degree of impropriety has been proposed in [8], where upper and lower bounds have been derived. Finally, it has been proved in [9] that two RVs with identical covariance matrix $\mathbf{R}_{z}=\mathrm{E}\left(\mathbf{z z}^{H}\right)$ and identical circularity coefficients can behave differently in second-order estimation and detection. Note that the results of this paper have already been partially presented in [10].

The aim of this correspondence is twofold. First, it aims in Section II to complement previously available theoretical results by introducing the non-circularity phase for scalar complex RV and attempts to extend it to multidimensional complex RVs with particular attention paid to rectilinear complex RVs. Second, since the knowledge of the circularity coefficients are generally crucial for using specific second-order algorithms dedicated to improper signals (e.g., in direction of arrival estimation, the specific algorithms [11], [12] dedicated to rectilinear sources fails when they are used for quasi rectilinear sources), the maximum likelihood (ML) estimate of the circularity coefficients in the Gaussian case and asymptotic distribution of this estimate for arbitrary distributions useful in practice for deriving confidence intervals are considered in Section III. We note that ML estimates have also been considered in [7]. However, the problem addressed was not the ML estimate of the circularity coefficients, but rather the GLRT for impropriety of complex signals where only the ML estimates of $\mathbf{R}_{z}$ and $\mathbf{R}_{z}^{\prime}$ are needed to derive the GLRT.

\section{RECTILINEAR COMPLEX RANDOM VARIABLE}

\section{A. Scalar complex random variable}

Let $z=x+i y$ denote a zero-mean second-order scalar complex $\mathrm{RV}$ with variance $\sigma_{z}^{2} \stackrel{\text { def }}{=} \mathrm{E}\left(|z|^{2}\right)$ and complementary variance $\mathrm{E}\left(z^{2}\right)$. The circularity coefficient $\rho$ of $z$ that is real-valued, non-negative and bounded above by 1 (see e.g., [6, lemma 4]) is defined by

$$
\rho e^{2 i \phi} \stackrel{\text { def }}{=} \frac{\mathrm{E}\left(z^{2}\right)}{\mathrm{E}\left(|z|^{2}\right)}
$$

where $\phi \in[0, \pi)$ is referred to as the non-circularity phase of $z$ in [13]. We note that this non-circularity phase has been introduced as a crucial parameter to study the statistical performance of MUSIC-like algorithms for estimating DOA of narrowband complex non-circular sources in [13] and then to characterize the resolution of closely spaced sources in [14].

If $\rho=0, z$ is referred to as proper in [15], [4] or circular to the second-order in [5], [6] and if $\rho=1, z$ is referred to as rectilinear in [16] (because in this case $z=|z| e^{i \phi}$ and $z$ lies in one line of $\mathcal{C}$ ) or most improper in [9]. If $\rho_{c o} \stackrel{\text { def }}{=} \frac{\mathrm{E}(x y)}{\sigma_{x} \sigma_{y}}$ with $\sigma_{x} \stackrel{\text { def }}{=} \sqrt{\mathrm{E}\left(x^{2}\right)}$ and $\sigma_{y} \stackrel{\text { def }}{=} \sqrt{\mathrm{E}\left(y^{2}\right)}$, denotes the correlation coefficient between the real $x$ and imaginary $y$ parts of $z$, the following relations between $\rho$ and $\rho_{c o}$ are proved in the Appendix ${ }^{1}$

\footnotetext{
${ }^{1}$ Note that there is a typo in the third property of Result 1 of [10].
} 
Result 1: The circularity coefficient $\rho$ of a scalar complex non degenerate $\left(\sigma_{x} \neq 0\right.$ and $\left.\sigma_{y} \neq 0\right)$ RV $z$ and the correlation coefficient $\rho_{c o}$ between its real $x$ and imaginary $y$ parts are related by the following relations

- $\rho=1 \Leftrightarrow \rho_{c o}= \pm 1$,

- $\rho=0 \Rightarrow \rho_{c o}=0$, the converse is false because $\rho_{c o}=0$ does not imply $\sigma_{x}=\sigma_{y}$,

- $\rho \geq\left|\rho_{c o}\right|$ and $\rho=\left|\rho_{c o}\right|$ when $\sigma_{x}=\sigma_{y}$.

To interpret the non-circularity phase $\phi$ of $z$, the following result is proved in the Appendix:

Result 2: For a non-circular scalar complex $\mathrm{RV} z$, the orthogonal regression line of the couple $(x, y)$ has a direction given by the non-circularity phase $\phi$ and the mean square orthogonal distance to this line is given ${ }^{2}$ by

$$
\mathrm{E}\left(d^{2}\right)=\frac{\sigma_{z}^{2}}{2}(1-\rho)
$$

Consequently, the larger $\rho$ is, the smaller the mean square distance of $(x, y)$ to the orthogonal regression line will be and this distance is zero if and only if $z$ is rectilinear along this orthogonal regression line whose direction is given by the non-circularity phase $\phi$.

\section{B. Multidimensional complex random variable}

Consider now a full $K$-dimensional zero-mean second-order complex $\mathrm{RV} \mathbf{z}=\mathbf{x}+i \mathbf{y}$ (i.e., with $\mathbf{R}_{z}$ nonsingular). The canonical correlations between $\mathbf{z}$ and $\mathbf{z}^{*}$ i.e., the circularity coefficients of $\mathbf{z}$, denoted by $\left(\rho_{k}\right)_{k=1, \ldots, K}$ has been defined in [6] and [7] as the singular values of the coherence matrix $\mathbf{M}=\mathbf{R}_{z}^{-1 / 2} \mathbf{R}_{z}^{\prime} \mathbf{R}_{z}^{-T / 2}$ associated with $\mathbf{z}$ and $\mathbf{z}^{*}$, that arranged in decreasing order satisfy $1=\rho_{1}=\ldots=\rho_{r}>\rho_{r+1} \geq \ldots \geq \rho_{K} \geq 0$ where $r$ denotes the number of circularity coefficients equal to 1 .

To attempt to extend the notion of non-circularity phase introduced in the scalar case, we first interpret the specific case $r=K$ introduced in [9] and referred to as most improper complex RVs z. Using [9, rel. (12)] and ([7, rel. (18)(19)] with $\mathbf{K}=\mathbf{I}$ ), it is straightforward to prove the following equivalences using the definitions $\tilde{\mathbf{z}} \stackrel{\text { def }}{=}\left[\mathbf{z}^{T}, \mathbf{z}^{H}\right]^{T}, \mathbf{w} \stackrel{\text { def }}{=}\left[\mathbf{x}^{T}, \mathbf{y}^{T}\right]^{T}, \mathbf{R}_{\tilde{z}} \stackrel{\text { def }}{=} \mathrm{E}\left(\tilde{\mathbf{z}} \tilde{\mathbf{z}}^{H}\right)$ and $\mathbf{R}_{w} \stackrel{\text { def }}{=} \mathrm{E}\left(\mathbf{w} \mathbf{w}^{T}\right)$

(i) the circularity spectrum is maximum, i.e., $\rho_{1}=\rho_{2}=\ldots=\rho_{K}=1$

(ii) $\operatorname{rank}\left(\mathbf{R}_{\tilde{z}}\right)=K$ (i.e., $\tilde{\mathbf{z}}$ belongs to a $K$-dimensional subspace of $\mathcal{C}^{2 K}$ ),

(iii) $\operatorname{rank}\left(\mathbf{R}_{w}\right)=K$ (i.e., w belongs to a $K$-dimensional subspace of $\mathcal{R}^{2 K}$ ),

(iv) there exists a square root $\mathbf{R}_{z}^{1 / 2}$ of $\mathbf{R}_{z}$ such that $\mathbf{R}_{z}^{\prime}=\mathbf{R}_{z}^{1 / 2} \mathbf{R}_{z}^{* / 2}$,

(v) there exist square roots $\mathbf{R}_{x}^{1 / 2}$ and $\mathbf{R}_{y}^{1 / 2}$ of $\mathbf{R}_{x}$ and $\mathbf{R}_{y}$ respectively, such that $\mathbf{R}_{x, y}=\mathbf{R}_{x}^{1 / 2} \mathbf{R}_{y}^{1 / 2}$.

${ }^{2}$ Note that the expression $\frac{\left(\sigma_{x}^{2}+\sigma_{y}^{2}\right)-\sqrt{\left(\sigma_{x}^{2}+\sigma_{y}^{2}\right)^{2}-4 \sigma_{x}^{2} \sigma_{y}^{2}\left(1-\rho_{c o}^{2}\right)}}{2}$ of this distance as a function of the correlation coefficient $\rho_{c o}$ given by the minimum eigenvalue of $\mathbf{R}_{w}$ is much involved.

${ }^{3}$ Note that the coherence matrix $\mathbf{M}$ depends on the specific square root $\mathbf{R}_{z}^{1 / 2}$ of $\mathbf{R}_{z}$, unique only if it is imposed to be positive definite Hermitian, in contrast to the circularity coefficients $\left(\rho_{1}, \ldots, \rho_{K}\right)$ which are always unique [6, th.2]. 
By analogy with the scalar case, we propose to call rectilinear such complex multidimensional RVs $\mathbf{z}$ whose circularity spectrum is maximum. Note that if the components $\left(z_{1}, \ldots, z_{K}\right)$ of $\mathbf{z}$ are all rectilinear, there are $K$ linear relations $y_{k}=\tan \left(\phi_{z_{k}}\right) x_{k},(k=1, . ., K)$ between the components of $\mathbf{w}$, consequently $\operatorname{rank}\left(\mathbf{R}_{w}\right)=K$ and $\mathbf{z}$ is rectilinear ${ }^{4}$. But the converse is not true: if $\mathbf{z}$ is rectilinear, its components $\left(z_{k}\right)_{k=1, . ., K}$ need not have maximum circularity coefficients $\rho_{z_{k}}$. For example, let $\mathbf{z}=\left(z_{1}, z_{2}\right)^{T}$ where $z_{1}$ is circular and $z_{2}=x_{2}+i y_{2}$ with $x_{2}=a x_{1}$ and $y_{2}=y_{1} . \mathbf{z}$ is rectilinear because $\mathbf{w}$ belongs to a 2-dimensional subspace of $\mathcal{R}^{4}$ but the circularity coefficients of $z_{1}$ and $z_{2}$ are $\rho_{z_{1}}=0$ and $\rho_{z_{2}}=\frac{\left|a^{2}-1\right|}{a^{2}+1}$.

To extend to the multidimensional case, the non-circularity phase $\phi$ defined in the scalar case by (1), we propose a definition based on the $K$-dimensional orthogonal regression subspace of $\left(x_{1}, \ldots, x_{K}, y_{1}, \ldots, y_{K}\right)$ which is the support of $\mathbf{w}$ for a maximum circularity spectrum. The canonical angles $\left(\phi_{1}, \phi_{2}, . ., \phi_{K^{2}}\right)$ between this subspace and each of the $K$ hyperspaces $\left(y_{k}=0\right)_{k=1, \ldots, K}$ of $\mathcal{R}^{2 K}$ satisfy this aim. However, two questions remain open. First, how to extend the expression (2) of the mean square orthogonal distance to this $K$-dimensional orthogonal regression subspace given in Result 2? Second, can on prove that the parameter $\left(\boldsymbol{\rho}, \boldsymbol{\phi}, \mathbf{R}_{z}\right)$ with $\boldsymbol{\phi} \stackrel{\text { def }}{=}\left(\phi_{1}, \phi_{2}, . ., \phi_{K^{2}}\right)^{T}$ makes up a one to one parametrization of $\left(\mathbf{R}_{z}, \mathbf{R}_{z}^{\prime}\right)$ ?

\section{ASYMPTOTIC DISTRIBUTION OF THE CIRCULARITY SPECTRUM ESTIMATE}

Let us consider the estimation of the circularity spectrum $\rho$ from $T$ independent identically distributed realizations $\left(\mathbf{z}_{t}\right)_{t=1, . ., T}$. The scalar and multidimensional cases are distinguished for the ease of the reader although the derivation mainly follows the same lines.

\section{A. Scalar complex random variable}

In the scalar-valued case, the following result is proved in the Appendix.

Result 3: When $z_{t}$ is Gaussian distributed, the maximum likelihood (ML) estimate $\left(\widehat{\rho}_{T}, \widehat{\phi}_{T}\right)$ of $(\rho, \phi)$ is given by $\left(\frac{\left|\sum_{t=1}^{T} z_{t}^{2}\right|}{\sum_{t=1}^{T}\left|z_{t}\right|^{2}}, \frac{1}{2} \operatorname{Arg}\left(\frac{\sum_{t=1}^{T} z_{t}^{2}}{\sum_{t=1}^{T}\left|z_{t}\right|^{2}}\right)\right)$. Furthermore, when $z_{t}$ is arbitrarily distributed with $\rho<1$, the sequence $\sqrt{T}\left(\widehat{\rho}_{T}-\rho\right)$ converges in distribution to the zero-mean Gaussian distribution of variance

$$
c_{\rho}=1-2 \rho^{2}+\rho^{4}+\rho^{2} \kappa+\frac{\kappa}{2}+\frac{\rho^{2} \Re\left(\kappa^{\prime}\right)}{2}-2 \rho^{2} \Re\left(\kappa^{\prime \prime}\right) \quad \text { if } c_{\rho} \neq 0,
$$

where $\kappa, \kappa^{\prime}$ and $\kappa^{\prime \prime}$ are the normalized-like cumulants $\frac{\operatorname{Cum}\left(z, z, z^{*}, z^{*}\right)}{\left(\mathrm{E}\left(|z|^{2}\right)\right)^{2}}, \frac{\operatorname{Cum}(z, z, z, z)}{\left(\mathrm{E}\left(z^{2}\right)\right)^{2}}$ and $\frac{\operatorname{Cum}\left(z, z, z, z^{*}\right)}{\mathrm{E}\left(|z|^{2}\right) \mathrm{E}\left(z^{2}\right)}$ respectively which are invariant to any rotation of the distribution of $z$.

Note that the variance $c_{\rho}$ of the asymptotic distribution of $\widehat{\rho}_{T}$ is a decreasing function $1-2 \rho^{2}+\rho^{4}$ of $\rho$ when $z_{t}$ is Gaussian distributed $\left(\kappa=\kappa^{\prime}=\kappa^{\prime \prime}=0\right)$. Furthermore, note that $c_{\rho} \leq 1-2 \rho^{2}+\rho^{4}$ is not valid for an arbitrary distribution of $z$ (in other words, the Gaussian case is not a worst case for the estimation of $\rho$ ). To be

\footnotetext{
${ }^{4}$ Note that the components $\left(z_{k}\right)_{k=1, . ., K}$ of $\mathbf{z}$ do not need to be uncorrelated as it is usually assumed in DOA estimation of non-circular sources (see e.g., [13], [11], [12]).
} 
convinced, consider $z \stackrel{\text { def }}{=} r e^{i \alpha}$ where $r$ and $\alpha$ are respectively Bernoulli $(p)$ and uniform on $[0,2 \pi)$ independent RVs. In this case, $z$ is zero-mean and circular to the second-order (i.e., $\rho=0$ ) and $\mathrm{E}\left(|z|^{4}\right)=\mathrm{E}\left(|z|^{2}\right)=p$. Consequently $\kappa=\frac{\mathrm{E}\left(|z|^{4}\right)-2\left(\mathrm{E}\left(|z|^{2}\right)\right)^{2}-\left|\mathrm{E}\left(z^{2}\right)\right|^{2}}{\left(\mathrm{E}\left(|z|^{2}\right)\right)^{2}}=\frac{1}{p}-2$ and $c_{0}=1+\frac{\kappa}{2}=\frac{1}{2 p}>1-2 \rho^{2}+\rho_{\mid \rho=0}^{4}$ for $p<\frac{1}{2}$.

In the particular case of rectilinear RVs for which $\rho=1$, we have $z_{t}=r_{t} e^{i \phi}$ with $r_{t}$ is a real-valued RV and with $\phi$ fixed. Consequently, the circularity coefficient and the non-circularity phase are perfectly estimated, i.e., $\widehat{\rho}_{T}=1$ and $\widehat{\phi}_{T}=\phi$. Besides in this case, $\kappa=\kappa^{\prime}=\kappa^{\prime \prime}$ are real-valued and we check from (3) that $c_{\rho}=0$ for arbitrary distribution. Furthermore, note that it is possible that $c_{\rho}=0$ with $\rho<1$ (such a situation will be illustrated in subsection III-C). In this case, the sequence $T\left(\widehat{\rho}_{T}-\rho\right)$ converges in distribution [20, Th.B, p. 124] to a Hermitian form $\mathbf{r}^{H} \mathbf{\Omega r}$, where $\mathbf{r}$ a two dimensional zero-mean complex Gaussian RV, whose distribution is defined by the right hand side of (7), and where our first order analysis does not allow one to specify the matrix $\Omega$.

\section{B. Multidimensional complex random variable}

In the multidimensional-valued case, the following result is proved in the Appendix.

Result 4: When $\mathbf{z}_{t}$ is Gaussian distributed, the ML estimate $\widehat{\boldsymbol{\rho}}_{T}$ of $\boldsymbol{\rho}=\left(\rho_{1}, \rho_{2}, \ldots, \rho_{K}\right)^{T}$ is given by the vector containing the $K$ singular values of the empirical coherence matrix $\mathbf{M}_{T}=\mathbf{R}_{z, T}^{-1 / 2} \mathbf{R}_{z, T}^{\prime} \mathbf{R}_{z, T}^{-T / 2}$ where $\mathbf{R}_{z, T} \stackrel{\text { def }}{=} \frac{1}{T} \sum_{t=1}^{T} \mathbf{z}_{t} \mathbf{z}_{t}^{H}$ and $\mathbf{R}_{z, T}^{\prime} \stackrel{\text { def }}{=} \frac{1}{T} \sum_{t=1}^{T} \mathbf{z}_{t} \mathbf{z}_{t}^{T}$. Furthermore, when $\mathbf{z}_{t}$ is arbitrarily distributed and when the circularity spectrum $\boldsymbol{\rho}$ has distinct elements, the sequence $\sqrt{T}\left(\widehat{\boldsymbol{\rho}}_{T}-\boldsymbol{\rho}\right)$ converges in distribution to a zero-mean Gaussian distribution that extends Result 3, whose covariance is given by

$$
\mathbf{C}_{\rho}=2 \Re\left(\mathbf{A}_{\rho} \mathbf{C}_{M} \mathbf{A}_{\rho}^{H}+\mathbf{A}_{\rho} \mathbf{C}_{M}^{\prime} \mathbf{A}_{\rho}^{T}\right)
$$

where ${ }^{5} \mathbf{A}_{\rho}=\frac{1}{2}(\mathbf{U} \circ \mathbf{U})^{H}$ with $\mathbf{U} \boldsymbol{\Delta} \mathbf{U}^{T}$ is the singular value decomposition (SVD) of the coherence matrix $\mathbf{M}$ and $\mathbf{C}_{M}$ and $\mathbf{C}_{M}^{\prime}$ are the two covariance matrices of the asymptotic distribution of the estimated coherence matrix $\mathbf{M}_{T}$ given by (12).

\section{Illustrative example}

Consider the baseband signal associated with a BPSK modulation. We assume no frequency offset, a sampling at the symbol rate and an inter-symbol interference of $P$ symbol, i.e.,

$$
z_{t}=\sum_{i=0}^{P} h_{i} s_{t-i} \quad \text { with } \quad s_{t} \in\{-1,+1\} \text { equiprobable and }\left(s_{t}\right)_{t=1,2, . .} \text { independent. }
$$

Naturally the sequence $s_{t}$ is rectilinear, but $z_{t}$ is no longer rectilinear, except for $h_{i}=a_{i} h_{0}, i=1, . ., P$ with $a_{i}$ real valued. We note that according to the values of $\left(h_{i}\right)_{i=0, . ., P}$, the circularity coefficient $\rho$ of $z_{t}$ can take arbitrary values in $[0,1]$. For example, $z_{t}$ becomes circular for $\sum_{i=0}^{P} h_{i}^{2}=0$.

\footnotetext{
${ }^{5} \mathbf{A} \circ \mathbf{B}$ denotes the Kharti-Rao matrix product (which is a column-wise Kronecker product, see e.g., [17]).
} 
Regarding the values of the asymptotic variance $c_{\rho}$ given by (3), we note that contrary to the Gaussian distribution, $\rho$ does not fix $c_{\rho}$. In particular $c_{\rho}=0$ and $c_{\rho}=1-2 \rho^{2}+\rho^{4}$ can be obtained for $\left(h_{i}\right)_{i=0, . ., P}$ as roots of polynomials in $\left(h_{i}, h_{i}^{*}\right)_{i=0, . ., P}$. For example, for $P=1$, it is straightforward to prove that

$$
\left(h_{0}, h_{1}\right)=\left(\sqrt{\frac{1+\rho}{2}}, i \sqrt{\frac{1-\rho}{2}}\right) \text { and }\left(h_{0}, h_{1}\right)=\left(\frac{1}{4} \sqrt{\rho+i \sqrt{1-\rho^{2}}}, \frac{1}{4} \sqrt{\rho-i \sqrt{1-\rho^{2}}}\right)
$$

gives $c_{\rho}=0$ and $c_{\rho}=1-2 \rho^{2}+\rho^{4}$ respectively, for arbitrary $\rho \in[0,1]$. Furthermore, we note that extensive numerical simulations show that $c_{\rho}$ is bounded above by its value associated with a Gaussian distribution, i.e.,

$$
c_{\rho} \leq 1-2 \rho^{2}+\rho^{4}
$$

This property seems valid for arbitrary channel impulse response $\left(h_{i}\right)_{i=0, . ., P}$, but we have not succeeded to prove it.

Through 500 realizations of a Rayleigh channel, Fig.1 validates (5) and consequently shows that the asymptotic accuracy of the estimate $\widehat{\rho}_{T}$ is always better for BPSK than for Gaussian symbols and arbitrary value of $\rho \in[0,1]$.

Fig.2 represents the asymptotic theoretical variance and empirical MSE of the estimate $\widehat{\rho}_{T}$, as a function of $T$ independent observations $z_{t}$ after decimating the original sequence $z_{t}$ by a factor of $P+1$, where $P$ is the memory of the simulated channel impulse response, for BPSK and Gaussian symbols for $P=1$ and $\rho=0.7$. More precisely for Gaussian symbols, $\left(h_{0}, h_{1}\right)=(0.921,0.387 i)$ with $c_{\rho}=0.260$ and for BPSK symbols $\left(h_{0}, h_{1}\right)=(0.710,0.492+0.502 i),\left(h_{0}, h_{1}\right)=(0.887,0.225+0.402 i)$ and $\left(h_{0}, h_{1}\right)=(0.921,0.387 i)$ are associated with $c_{\rho}=0.260, c_{\rho}=0.084$ and $c_{\rho}=0$ respectively. We see that the domain of validity of our asymptotic analysis for $c_{p} \neq 0$ is very large $(T>100)$. Furthermore, we see that for $c_{p}=0$, the empirical MSE varies in $1 / T^{2}$ in contrast to $c_{p} \neq 0$, for which the asymptotic theoretical variance is $c_{\rho} / T$.

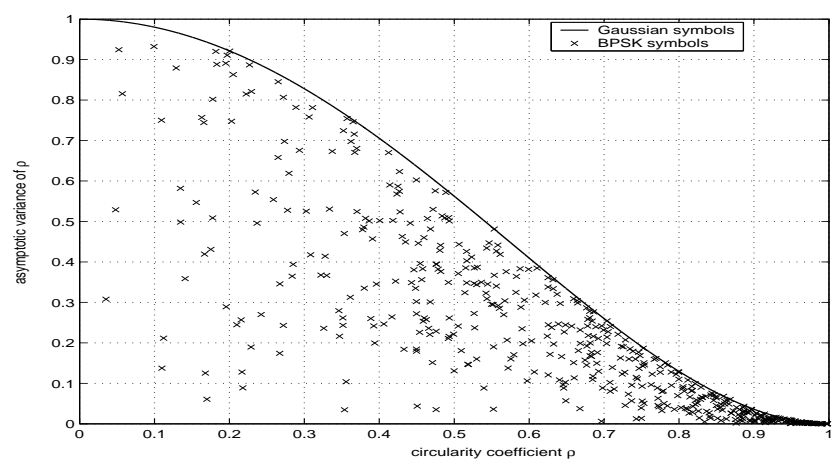

Fig.1 Asymptotic theoretical variance $c_{\rho}$ given by (3) as a function of the circularity coefficient $\rho$ for BPSK and Gaussian symbols $s_{t}$ for $P=2$ and 500 realizations of a Rayleigh channel whose three coefficients have same variance.

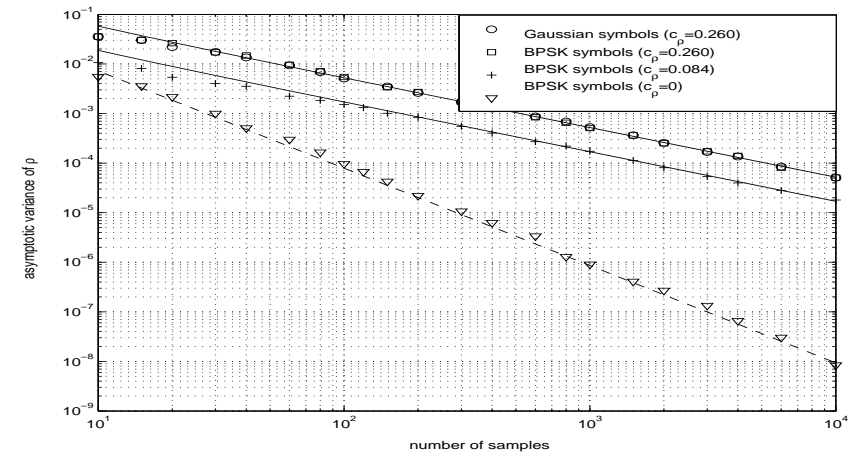

Fig.2 Asymptotic theoretical variance and empirical (averaging 1000 runs) MSE of the estimate $\widehat{\rho}_{T}$, compared to the asymptotic theoretical variance $c_{\rho}$ as a function of the number $T$ of observations for Gaussian and BPSK symbols associated with different channels $\left(h_{0}, h_{1}\right)$ for $\rho=0.7$. 


\section{APPENDIX}

\section{Proof of Result 1}

From the following expression of the circularity coefficient:

$$
\rho=\sqrt{\frac{\left(\frac{\sigma_{x}}{\sigma_{y}}-\frac{\sigma_{y}}{\sigma_{x}}\right)^{2}}{\left(\frac{\sigma_{x}}{\sigma_{y}}+\frac{\sigma_{y}}{\sigma_{x}}\right)^{2}}+4 \rho_{c o}^{2} \frac{1}{\left(\frac{\sigma_{x}}{\sigma_{y}}+\frac{\sigma_{y}}{\sigma_{x}}\right)^{2}}}
$$

the first two bullets are straightforwardly proved.

For the third one, $\rho^{2}=1-\frac{4\left(1-\rho_{c o}^{2}\right)}{\left(\frac{\sigma x}{\sigma_{y}}+\frac{\sigma_{y}}{\sigma_{x}}\right)^{2}} \geq \rho_{c o}^{2}$ because $\frac{\sigma_{x}}{\sigma_{y}}+\frac{\sigma_{y}}{\sigma_{x}} \geq 2$.

\section{Proof of Result 2}

The orthogonal regression line (see e.g., [18]) of the couple $(x, y)$ is given by the line orthogonal to the eigenvector $\mathbf{u}$ associated with the minimum eigenvalue $\lambda$ of the covariance matrix $\mathbf{R}_{w}$ of $\mathbf{w} \stackrel{\text { def }}{=}[x, y]^{T}$ and the mean square orthogonal distance $\mathrm{E}\left(d^{2}\right)$ to this line is given by $\lambda$. To solve easily this problem, it is convenient to work with the augmented vector $\tilde{\mathbf{z}} \stackrel{\text { def }}{=}\left[z, z^{*}\right]^{T}$ whose covariance matrix $\mathbf{R}_{\tilde{z}}$ is related to $\mathbf{R}_{w}$ by $\mathbf{R}_{w}=\frac{1}{2} \mathbf{T}^{H} \mathbf{R}_{\tilde{z}} \mathbf{T}$ using $\tilde{\mathbf{z}}=\sqrt{2} \mathbf{T w}$, where $\mathbf{T}$ is the unitary matrix $\frac{1}{\sqrt{2}}\left(\begin{array}{cc}1 & i \\ 1 & -i\end{array}\right)$. Because the minimum eigenvalue and the associated unit eigenvector of $\mathbf{R}_{\tilde{z}}=\sigma_{z}^{2}\left(\begin{array}{cc}1 & \rho e^{2 i \phi} \\ \rho e^{-2 i \phi} & 1\end{array}\right)$ are $\lambda=\sigma_{z}^{2}(1-\rho)$ and $\mathbf{u}=\frac{i}{\sqrt{2}}\left[e^{i \phi},-e^{-i \phi}\right]^{T}$, the minimum eigenvalue and the associated unit eigenvector of $\mathbf{R}_{w}$ are $\frac{1}{2} \lambda$ and $\mathbf{T}^{H} \mathbf{u}=[-\sin \phi, \cos \phi]^{T} \perp[\cos \phi, \sin \phi]^{T}$.

\section{Proof of Result 3}

When $z_{t}$ is Gaussian distributed, the log-likelihood function associated with $\left(z_{t}\right)_{t=1, . ., T}$ can be classically written after dropping the constants as

$$
L\left(\rho, \phi, \sigma_{z}^{2}\right)=-\frac{T}{2}\left(\ln \left[\operatorname{Det}\left(\mathbf{R}_{\tilde{z}}\right)\right]+\operatorname{Tr}\left(\mathbf{R}_{\tilde{z}}^{-1} \mathbf{R}_{\tilde{z}, T}\right)\right)
$$

with $\mathbf{R}_{\tilde{z}, T} \stackrel{\text { def }}{=} \frac{1}{T} \sum_{t=1}^{T} \tilde{\mathbf{z}}_{t} \tilde{\mathbf{z}}_{t}^{H}$, where the parameter $\left(\rho, \phi, \sigma_{z}^{2}\right)$ is embedded in the covariance matrix $\mathbf{R}_{\tilde{z}}$. Due to the structure $\left[\begin{array}{cc}(\times) & (\diamond) \\ (\diamond)^{*} & (\times)^{*}\end{array}\right]$ of $\mathbf{R}_{\tilde{z}}$, the ML estimation of $\mathbf{R}_{\tilde{z}}$ becomes a constrained optimization problem which is not standard. But maximizing the log-likelihood (6) without any constraint on the Hermitian matrix $\mathbf{R}_{\tilde{z}}$ reduces to a standard maximization problem, whose solution is $\mathbf{R}_{\tilde{z}, T}$. Because $\mathbf{R}_{\tilde{z}, T}=\left[\begin{array}{cc}\frac{1}{T} \sum_{t=1}^{T}\left|z_{t}\right|^{2} & \frac{1}{T} \sum_{t=1}^{T} z_{t}^{2} \\ \frac{1}{T} \sum_{t=1}^{T} z_{t}^{* 2} & \frac{1}{T} \sum_{t=1}^{T}\left|z_{t}\right|^{2}\end{array}\right]$ is also structured as $\left[\begin{array}{cc}(\times) & (\diamond) \\ (\diamond)^{*} & (\times)^{*}\end{array}\right], \mathbf{R}_{\tilde{z}, T}$ is the ML estimate of $\mathbf{R}_{\tilde{z}}$. Using the invariance property of the ML estimate implies that the ML estimate of $(\rho, \phi)$ is given by $\left(\frac{\left|\sum_{t=1}^{T} z_{t}^{2}\right|}{\sum_{t=1}^{T}\left|z_{t}\right|^{2}}, \frac{1}{2} \operatorname{Arg}\left(\frac{\sum_{t=1}^{T} z_{t}^{2}}{\sum_{t=1}^{T}\left|z_{t}\right|^{2}}\right)\right)$.

Deriving the asymptotic distribution of the empirical estimate $\widehat{\rho}_{T}$ when $z_{t}$ is arbitrarily distributed, relies on 
the standard central limit theorem ${ }^{6}$ applied to the independent identically distributed bidimensional complex $\operatorname{RVs}\left(\begin{array}{c}r_{z, T} \\ r_{z, T}^{\prime}\end{array}\right)$ with $r_{z, T}=\frac{1}{T} \sum_{t=1}^{T}\left|z_{t}^{2}\right|$ and $r_{z, T}^{\prime}=\frac{1}{T} \sum_{t=1}^{T} z_{t}^{2}$ :

$$
\sqrt{T}\left(\begin{array}{c}
r_{z, T}-r_{z} \\
r_{z, T}^{\prime}-r_{z}^{\prime}
\end{array}\right) \stackrel{\mathcal{L}}{\rightarrow} \mathcal{N}_{C}\left(\left(\begin{array}{l}
0 \\
0
\end{array}\right),\left(\begin{array}{cc}
c_{r} & c_{r, r^{\prime}} \\
c_{r^{\prime}, r} & c_{r^{\prime}}
\end{array}\right),\left(\begin{array}{cc}
c_{r}^{\prime} & c_{r, r^{\prime}}^{\prime} \\
c_{r^{\prime}, r}^{\prime} & c_{r^{\prime}}^{\prime}
\end{array}\right)\right)
$$

where $r_{z}=\mathrm{E}\left|z_{t}^{2}\right|=\sigma_{z}^{2}, r_{z}^{\prime}=\mathrm{E}\left(z_{t}^{2}\right)=\rho \sigma_{z}^{2} e^{i 2 \phi}$ and where $\left(\begin{array}{cc}c_{r} & c_{r, r^{\prime}} \\ c_{r^{\prime}, r} & c_{r^{\prime}}\end{array}\right)$ and $\left(\begin{array}{cc}c_{r}^{\prime} & c_{r, r^{\prime}}^{\prime} \\ c_{r^{\prime}, r}^{\prime} & c_{r^{\prime}}^{\prime}\end{array}\right)$ denote the covariance and the complementary covariance matrices of the bidimensional RV $\left(\left|z_{t}^{2}\right|, z_{t}^{2}\right)^{T}$. Using the identity

$$
\mathrm{E}\left(z_{1} z_{2} z_{3} z_{4}\right)=\mathrm{E}\left(z_{1} z_{2}\right) \mathrm{E}\left(z_{3} z_{4}\right)+\mathrm{E}\left(z_{1} z_{3}\right) \mathrm{E}\left(z_{2} z_{4}\right)+\mathrm{E}\left(z_{1} z_{4}\right) \mathrm{E}\left(z_{2} z_{3}\right)+\operatorname{Cum}\left(z_{1}, z_{2}, z_{3}, z_{4}\right)
$$

we straightforwardly obtain

$$
\begin{aligned}
& \left(\begin{array}{cc}
c_{r} & c_{r, r^{\prime}} \\
c_{r^{\prime}, r} & c_{r^{\prime}}
\end{array}\right)=\sigma_{z}^{4}\left(\begin{array}{cc}
1+\rho^{2}+\kappa & \rho e^{-i 2 \phi}\left(2+\kappa^{\prime \prime *}\right) \\
\rho e^{i 2 \phi}\left(2+\kappa^{\prime \prime}\right) & 2+\kappa
\end{array}\right) \\
& \left(\begin{array}{cc}
c_{r}^{\prime} & c_{r, r^{\prime}}^{\prime} \\
c_{r^{\prime}, r}^{\prime} & c_{r^{\prime}}^{\prime}
\end{array}\right)=\sigma_{z}^{4}\left(\begin{array}{cc}
1+\rho^{2}+\kappa & \rho e^{i 2 \phi}\left(2+\kappa^{\prime \prime}\right) \\
\rho e^{i 2 \phi}\left(2+\kappa^{\prime \prime}\right) & \rho^{2} e^{i 4 \phi}\left(2+\kappa^{\prime}\right)
\end{array}\right) .
\end{aligned}
$$

Then, considering the following mappings

$$
\left(r_{z, T}, r_{z, T}^{\prime}\right) \longmapsto m_{T}=\frac{r_{z, T}^{\prime}}{r_{z, T}} \longmapsto \widehat{\rho}_{T}=\sqrt{m_{T} m_{T}^{*}}
$$

with their associated differentials

$$
d m=-\frac{r^{\prime}}{r^{2}} d r+\frac{1}{r} d r^{\prime} \quad \text { and } \quad d \rho=\frac{1}{2 \rho}\left(m^{*} d m+m d m^{*}\right)
$$

the standard theorem of continuity (see e.g., [20, Th.A, p. 122]) on regular functions of asymptotically Gaussian statistics applies. Consequently, we obtain the following convergence in distribution with $m=\frac{r_{z}^{\prime}}{r_{z}}=\rho e^{i 2 \phi}$

$$
\sqrt{T}\left(m_{T}-m\right) \stackrel{\mathcal{L}}{\rightarrow} \mathcal{N}_{C}\left(0, c_{m}, c_{m}^{\prime}\right)
$$

where

$$
c_{m}=\left(\begin{array}{cc}
-\frac{r_{z}^{\prime}}{r_{z}^{2}} & \frac{1}{r_{z}}
\end{array}\right)\left(\begin{array}{cc}
c_{r} & c_{r, r^{\prime}} \\
c_{r^{\prime}, r} & c_{r^{\prime}}
\end{array}\right)\left(\begin{array}{c}
-\frac{r_{z}^{\prime}}{r_{z}^{2}} \\
\frac{1}{r_{z}}
\end{array}\right), \quad c_{m}^{\prime}=\left(\begin{array}{cc}
-\frac{r_{z}^{\prime}}{r_{z}^{2}} & \frac{1}{r_{z}}
\end{array}\right)\left(\begin{array}{cc}
c_{r}^{\prime} & c_{r, r^{\prime}}^{\prime} \\
c_{r^{\prime}, r}^{\prime} & c_{r^{\prime}}^{\prime}
\end{array}\right)\left(\begin{array}{c}
-\frac{r_{z}^{\prime}}{r_{z}^{2}} \\
\frac{1}{r_{z}}
\end{array}\right)
$$

${ }^{6} \stackrel{\mathcal{L}}{\rightarrow}$ means the convergence in distribution when $T \rightarrow \infty$, while $\mathcal{N}_{R}(\mathbf{m}, \mathbf{C})$ and $\mathcal{N}_{C}\left(\mathbf{m}, \mathbf{C}, \mathbf{C}^{\prime}\right)$ denote Gaussian real and complex distribution with mean, covariance and complementary covariance are $\mathbf{m}, \mathbf{C}$ and $\mathbf{C}^{\prime}$ respectively. 
and

$$
\sqrt{T}\left(\widehat{\rho}_{T}-\rho\right) \stackrel{\mathcal{L}}{\rightarrow} \mathcal{N}_{R}\left(0, c_{\rho}\right),
$$

where $c_{\rho}=\frac{1}{4 \rho^{2}}\left(\begin{array}{cc}m^{*} & m\end{array}\right)\left(\begin{array}{cc}c_{m} & c_{m}^{\prime} \\ c_{m}^{\prime}{ }^{*} & c_{m}{ }^{*}\end{array}\right)\left(\begin{array}{c}m \\ m^{*}\end{array}\right)=\frac{1}{2}\left(c_{m}+\Re\left(c_{m}^{\prime} e^{-4 i \phi}\right)\right)$. Inserting (9) and (10) into expressions (11) of $c_{m}$ and $c_{m}^{\prime}$, (3) follows thanks to simple algebraic manipulations.

When $z$ is replaced with $z e^{i \alpha}$ with $\alpha$ fixed, using the multilinearity of $\operatorname{Cum}\left(z_{1}, z_{2}, z_{3}, z_{4}\right)$, the normalizedlike cumulants $\kappa, \kappa^{\prime}$ and $\kappa^{\prime \prime}$ are invariant, so $c_{p}$ as well, whereas the non-circularity phase $\phi$ is replaced by $\phi+\alpha$.

\section{Proof of Result 4}

The proof follows the same steps that for Result 3. When $\mathbf{z}_{t}$ is Gaussian distributed, the log-likelihood function $L\left(\boldsymbol{\rho}, \phi, \mathbf{R}_{z}\right)$ has form (6) and consequently $\mathbf{R}_{\tilde{z}, T}$ is the ML estimate of $\mathbf{R}_{\tilde{z}}$. Using the SVD of the coherence matrix [21, 4.4.4] $\mathbf{M}=\mathbf{U} \boldsymbol{\Delta} \mathbf{U}^{T}=\mathbf{R}_{z}^{-1 / 2} \mathbf{R}_{z}^{\prime} \mathbf{R}_{z}^{-T / 2}$, the invariance property of the ML implies that the ML estimate of $\boldsymbol{\rho}$ is given by the $K$ th vector $\operatorname{diag}\left(\boldsymbol{\Delta}_{T}\right)$ containing the $K$ singular values of the empirical coherence matrix $\mathbf{M}_{T}=\mathbf{R}_{z, T}^{-1 / 2} \mathbf{R}_{z, T}^{\prime} \mathbf{R}_{z, T}^{-T / 2}$.

Deriving the asymptotic distribution of the empirical estimate $\widehat{\boldsymbol{\rho}}_{T}$ when $\mathbf{z}_{t}$ is arbitrarily distributed is based on the following mapping

$$
\left(\mathbf{R}_{z, T}, \mathbf{R}_{z, T}^{\prime}\right) \longmapsto \mathbf{M}_{T} \stackrel{\text { def }}{=} \mathbf{R}_{z, T}^{-1 / 2} \mathbf{R}_{z, T}^{\prime} \mathbf{R}_{z, T}^{-T / 2}=\mathbf{U}_{T} \boldsymbol{\Delta}_{T} \mathbf{U}_{T}^{T} \longmapsto \widehat{\boldsymbol{\rho}}_{T} \stackrel{\text { def }}{=} \operatorname{diag}\left(\boldsymbol{\Delta}_{T}\right) .
$$

Thus, the first step consists in deriving the asymptotic distribution of $\left(\mathbf{R}_{z, T}, \mathbf{R}_{z, T}^{\prime}\right)$ given by the standard central limit theorem:

$$
\sqrt{T}\left(\operatorname{vec}\left(\mathbf{R}_{z, T}, \mathbf{R}_{z, T}^{\prime}\right)-\operatorname{vec}\left(\mathbf{R}_{z}, \mathbf{R}_{z}^{\prime}\right)\right) \stackrel{\mathcal{L}}{\rightarrow} \mathcal{N}_{C}\left(\mathbf{0},\left(\begin{array}{cc}
\mathbf{C}_{R} & \mathbf{C}_{R, R^{\prime}} \\
\mathbf{C}_{R^{\prime}, R} & \mathbf{C}_{R^{\prime}}
\end{array}\right),\left(\begin{array}{cc}
\mathbf{C}_{R}^{\prime} & \mathbf{C}_{R, R^{\prime}}^{\prime} \\
\mathbf{C}_{R^{\prime}, R}^{\prime} & \mathbf{C}_{R^{\prime}}^{\prime}
\end{array}\right)\right),
$$

where the expressions of the different covariance matrices are deduced from identity (8). For example

$$
\begin{aligned}
\left(\mathbf{C}_{R}\right)_{i+(j-1) K, k+(l-1) K} & =\mathrm{E}\left(z_{t, i} z_{t, j}^{*} z_{t, k}^{*} z_{t, l}\right)-\mathrm{E}\left(z_{t, i} z_{t, j}^{*}\right) \mathrm{E}\left(z_{t, k}^{*} z_{t, l}\right) \\
& =\mathrm{E}\left(z_{t, i} z_{t, k}^{*}\right) \mathrm{E}\left(z_{t, j}^{*} z_{t, l}\right)+\mathrm{E}\left(z_{t, i} z_{t, l}\right) \mathrm{E}\left(z_{t, j}^{*} z_{t, k}^{*}\right)+\operatorname{Cum}\left(z_{t, i}, z_{t, j}^{*}, z_{t, k}^{*}, z_{t, l}\right)
\end{aligned}
$$

which gives $\mathbf{C}_{R}=\mathbf{R}_{z}^{*} \otimes \mathbf{R}_{z}+\mathbf{K}\left(\mathbf{R}_{z}^{\prime} \otimes \mathbf{R}_{z}^{*}\right)+\mathbf{Q}_{z}$ where $\left(\mathbf{Q}_{z}\right)_{i+(j-1) K, k+(l-1) K}=\operatorname{Cum}\left(z_{t, i}, z_{t, j}^{*}, z_{t, k}^{*}, z_{t, l}\right)$ and where $\otimes$ denotes the standard Kronecker product of matrices and $\mathbf{K}$ the vec-permutation matrix which transforms $\operatorname{vec}(\mathbf{C})$ to $\operatorname{vec}\left(\mathbf{C}^{T}\right)$ for any square matrix $\mathbf{C}$. The other covariance matrices are deduced in the same 
way (see e.g., [3]) and are given by

$$
\begin{aligned}
\mathbf{C}_{R^{\prime}} & =\mathbf{R}_{z} \otimes \mathbf{R}_{z}+\mathbf{K}\left(\mathbf{R}_{z} \otimes \mathbf{R}_{z}\right)+\mathbf{Q}_{z}^{\prime} \\
\mathbf{C}_{R}^{\prime} & =\mathbf{C}_{R} \mathbf{K} \\
\mathbf{C}_{R^{\prime}}^{\prime} & =\mathbf{R}_{z}^{\prime} \otimes \mathbf{R}_{z}^{\prime}+\mathbf{K}\left(\mathbf{R}_{z}^{\prime} \otimes \mathbf{R}_{z}^{\prime}\right)+\mathbf{Q}_{z}^{\prime \prime} \\
\mathbf{C}_{R, R^{\prime}} & =\mathbf{C}_{R^{\prime}, R}^{H}=\mathbf{R}_{z}^{\prime *} \otimes \mathbf{R}_{z}+\mathbf{K}\left(\mathbf{R}_{z} \otimes \mathbf{R}_{z}^{\prime *}\right)+\mathbf{Q}_{z}^{\prime \prime \prime} \\
\mathbf{C}_{R, R^{\prime}}^{\prime} & =\mathbf{C}_{R^{\prime}, R}^{\prime T}=\mathbf{R}_{z}^{\prime} \otimes \mathbf{R}_{z}^{*}+\mathbf{K}\left(\mathbf{R}_{z}^{\prime} \otimes \mathbf{R}_{z}^{*}\right)+\mathbf{Q}_{z}^{\prime \prime \prime \prime}
\end{aligned}
$$

where $\left(\mathbf{Q}_{z}^{\prime}\right)_{i+(j-1) K, k+(l-1) K}=\operatorname{Cum}\left(z_{t, i}, z_{t, j}, z_{t, k}^{*}, z_{t, l}^{*}\right),\left(\mathbf{Q}_{z}^{\prime \prime}\right)_{i+(j-1) K, k+(l-1) K}=\operatorname{Cum}\left(z_{t, i}, z_{t, j}, z_{t, k}, z_{t, l}\right)$, $\left(\mathbf{Q}_{z}^{\prime \prime \prime}\right)_{i+(j-1) K, k+(l-1) K}=\operatorname{Cum}\left(z_{t, i}, z_{t, j}^{*}, z_{t, k}^{*}, z_{t, l}^{*}\right)$ and $\left(\mathbf{Q}_{z}^{\prime \prime \prime \prime}\right)_{i+(j-1) K, k+(l-1) K}=\operatorname{Cum}\left(z_{t, i}, z_{t, j}^{*}, z_{t, k}, z_{t, l}\right)$.

With the differential of the mapping $\left(\mathbf{R}_{z, T}, \mathbf{R}_{z, T}^{\prime}\right) \longmapsto \mathbf{M}_{T}=\mathbf{R}_{z, T}^{-1 / 2} \mathbf{R}_{z, T}^{\prime} \mathbf{R}_{z, T}^{-T / 2}$

$$
\begin{aligned}
\operatorname{vec}(d \mathbf{M}) & =-\left(\left(\mathbf{R}_{z}^{-1 / 2} \mathbf{R}_{z}^{T} \otimes \mathbf{I}\right)+\left(\mathbf{I} \otimes \mathbf{R}_{z}^{-1 / 2} \mathbf{R}_{z}^{\prime}\right)\right)\left(\left(\mathbf{R}_{z}^{-T / 2} \otimes \mathbf{I}\right)+\left(\mathbf{I} \otimes \mathbf{R}_{z}^{-1 / 2}\right)\right)^{-1}\left(\mathbf{R}_{z}^{-T} \otimes \mathbf{R}_{z}^{-1}\right) \operatorname{vec}\left(d \mathbf{R}_{z}\right) \\
& +\left(\mathbf{R}_{z}^{-T / 2} \otimes \mathbf{R}_{z}^{-1 / 2}\right) \operatorname{vec}\left(d \mathbf{R}_{z}^{\prime}\right) \stackrel{\text { def }}{=} \mathbf{A}_{r} \operatorname{vec}\left(d\left(\mathbf{R}_{z}\right)+\mathbf{A}_{r^{\prime}} \operatorname{vec}\left(d \mathbf{R}_{z}^{\prime}\right)\right.
\end{aligned}
$$

we obtain the following convergence in distribution by the standard theorem of continuity (see e.g., [20, Th.A, p. 122])

$$
\sqrt{T}\left(\operatorname{vec}\left(\mathbf{M}_{T}\right)-\operatorname{vec}(\mathbf{M})\right) \stackrel{\mathcal{L}}{\rightarrow} \mathcal{N}_{C}\left(\mathbf{0}, \mathbf{C}_{M}, \mathbf{C}_{M}^{\prime}\right),
$$

where $\mathbf{C}_{M}=\mathbf{A}_{r} \mathbf{C}_{R} \mathbf{A}_{r}^{H}+\mathbf{A}_{r} \mathbf{C}_{R, R^{\prime}} \mathbf{A}_{r^{\prime}}^{H}+\mathbf{A}_{r^{\prime}} \mathbf{C}_{R^{\prime} R} \mathbf{A}_{r}^{H}+\mathbf{A}_{r^{\prime}} \mathbf{C}_{R^{\prime}} \mathbf{A}_{r^{\prime}}^{H}$ and $\mathbf{C}_{M}^{\prime}=\mathbf{A}_{r} \mathbf{C}_{R}^{\prime} \mathbf{A}_{r}^{T}+\mathbf{A}_{r} \mathbf{C}_{R, R^{\prime}}^{\prime} \mathbf{A}_{r^{\prime}}^{T}+$ $\mathbf{A}_{r^{\prime}} \mathbf{C}_{R^{\prime} R}^{\prime} \mathbf{A}_{r}^{T}+\mathbf{A}_{r^{\prime}} \mathbf{C}_{R^{\prime}}^{\prime} \mathbf{A}_{r^{\prime}}^{T}$.

Finally, consider the mapping $\mathbf{M}_{T} \longmapsto \widehat{\boldsymbol{\rho}}_{T}=\operatorname{diag}\left(\boldsymbol{\Delta}_{T}\right)$. Noting that the eigenvalues of $\mathbf{M}_{T} \mathbf{M}_{T}^{H}$ are the squares of the singular values of $\mathbf{M}_{T}$, the differential of the simple eigenvalues of $\mathbf{M}_{T} \mathbf{M}_{T}^{H}$ gives (see e.g., $[22$, th.8, ch.9])

$$
d\left(\rho_{k}^{2}\right)=\mathbf{u}_{k}^{H} d\left(\mathbf{M M}^{H}\right) \mathbf{u}_{k}
$$

where $\mathbf{u}_{k}$ denotes the left singular vector of $\mathbf{M}$ associated with the singular value $\rho_{k}$. Using $\mathbf{M}^{H} \mathbf{u}_{k}=\rho_{k} \mathbf{u}_{k}^{*}$, (13) gives $d \rho_{k}=\frac{1}{2}\left(\mathbf{u}_{k}^{H} d \mathbf{M} \mathbf{u}_{k}^{*}+\mathbf{u}_{k}^{T} d \mathbf{M}^{H} \mathbf{u}_{k}^{*}\right)$, and more compactly

$$
d \boldsymbol{\rho}=\frac{1}{2}\left((\mathbf{U} \circ \mathbf{U})^{H} \operatorname{vec}(d \mathbf{M})+(\mathbf{U} \circ \mathbf{U})^{T} \operatorname{vec}\left(d \mathbf{M}^{*}\right)\right) \stackrel{\text { def }}{=} \mathbf{A}_{\rho} \operatorname{vec}(d \mathbf{M})+\mathbf{A}_{\rho}^{*} \operatorname{vec}\left(d \mathbf{M}^{*}\right) .
$$

Consequently, the covariance matrix of the asymptotic Gaussian distribution of $\widehat{\boldsymbol{\rho}}_{T}$ is given by (4).

\section{REFERENCES}

[1] B. Picinbono and P. Chevalier "Widely Linear Estimation with Complex Data," IEEE Trans. Signal Process., vol. 43, no. 8, pp. 2030-2033, August 1995. 
[2] P.J. Schreier, L. Scharf and C.T. Mullis, "Detection and estimation of improper complex random signals," IEEE Trans. Inform. Theory, vol. 51, no. 1, pp. 306-312, January 2005.

[3] J.P. Delmas, "Asymptotically minimum variance second-order estimation for non-circular signals with application to DOA estimation," IEEE Trans. Signal Process., vol. 52, no. 5, pp. 1235-1241, May 2004.

[4] P.J. Schreier and L. Scharf, "Second-order Analysis of improper complex random vectors and processes," IEEE Trans. Signal Process., vol. 51, no. 3, pp. 714-725, March 2003.

[5] B. Picinbono, "Second-order complex random vectors and normal distributions," IEEE Trans. Signal Process., vol. 44, no. 10, pp. 2637-2640, October 1996.

[6] J. Eriksson and V. Koivunen, "Complex random vectors and ICA models: identifiability, uniqueness, and separability," IEEE Trans. Inform. Theory, vol. 52 no. 3, pp. 1017-1029, March 2006.

[7] P.J. Schreier, L. Scharf and A. Hanssen, "A generalized likelihood ratio test for impropriety of complex signals," IEEE Signal Process. Letters, vol. 13, no. 7, pp. 433-436, July 2006.

[8] P.J. Schreier, "Bound on the degree of improperty of complex random vectors," IEEE Signal Process. Letters, vol. 15, pp. 190-193, 2008.

[9] P.J. Schreier, "The degree of improperty (noncircularity) of complex random vectors," Proc. ICASSP, Las Vegas, April 2008.

[10] H. Abeida and J.P. Delmas, "On the degree of second-order non-circularity of complex random variables", Proc. ICASSP, Las Vegas, April 2008.

[11] P. Chargé, Y. Wang and J. Saillard, "A non-circular sources direction finding method using polynomial rooting," Signal Processing, vol. 81, pp. 1765-1770, 2001.

[12] M. Haardt and F. Römer, "Enhancements of unitary ESPRIT for non circular sources," International Conference on ASSP, Montreal, May 2004.

[13] H. Abeida and J.P. Delmas, "MUSIC-like estimation of direction of arrival for non-circular sources," IEEE Trans. Signal Process., vol. 54, no. 7, pp. 2678-2690, July 2006.

[14] H. Abeida, J.P. Delmas, "Statistical performance of MUSIC-like algorithms in resolving noncircular sources," IEEE Trans on Signal Processing, vol. 56, no. 9, pp. 4317-4329, September 2008.

[15] F.D. Neeser and J.L. Massey, "Proper complex random proceses with applications to information theory," IEEE Trans. Inform. Theory, vol. 39 no. 4, pp. 1293-1302, July 1993.

[16] P. Chevalier and F. Pipon, "New insights into optimal widely linear array receivers for the demodulation of BPSK, MSK and GMSK signals corrupted by non circular interferences - Application to SAIC", IEEE Trans. Signal Process., vol. 54, no. 3, pp. 870-883, March 2006.

[17] J.W. Brewer, "Kronecker products and matrix calculus in system theory," IEEE Trans. Circuits System, vol. CAS-25, pp. 772-781, September 1978.

[18] I.T. Jolliffe, Principal Component Analysis Series: Springer Series in Statistics, 2002.

[19] D.A. Harville, Matrix algebra from a statistician's perspective, Springer 1999.

[20] R.J. Serfling, Approximation Theorems of Mathematical Statistics, John Wiley and Sons, 1980.

[21] R.A Horn and C.R. Johnson,, Matrix analysis, Cambridge Univer sity Press, 1985.

[22] J.R. Magnus and H. Neudecker, Matrix differential calculus with applications in statistics and econometrics, Wiley series in probability and statistics, revised edition, 1999. 